Draft Version OCtOBER 27, 2018

Preprint typeset using $\mathrm{LAT}_{\mathrm{E}} \mathrm{X}$ style emulateapj v. 11/10/09

\title{
DIFFUSE TIDAL STRUCTURES IN THE HALOS OF VIRGO ELLIPTICALS
}

\author{
Steven Janowiecki, ${ }^{1,2}$ J. Christopher Mihos, ${ }^{1}$ Paul Harding, ${ }^{1}$ John J. Feldmeier, ${ }^{3}$ \\ Craig Rudick, ${ }^{1}$ and Heather Morrison ${ }^{1}$ \\ (Received 2010 March 16; Accepted 2010 April 7; Published 2010 May 5) \\ Draft version October 27, 2018
}

\begin{abstract}
We use deep V-band surface photometry of five of the brightest elliptical galaxies in the Virgo cluster to search for diffuse tidal streams, shells, and plumes in their outer halos $(r>50 \mathrm{kpc})$. We fit and subtract elliptical isophotal models from the galaxy images to reveal a variety of substructure, with surface brightnesses in the range $\mu_{\mathrm{V}}=26-29 \mathrm{mag} / \operatorname{arcsec}^{2}$. M49 possesses an extended, interleaved shell system reminiscent of the radial accretion of a satellite companion, while M89's complex system of shells and plumes suggests a more complicated accretion history involving either multiple events or a major merger. M87 has a set of long streamers as might be expected from stripping of low luminosity dwarfs on radial orbits in Virgo. M86 also displays a number of small streams indicative of stripping of dwarf companions, but these comprise much less luminosity than those of M87. Only M84 lacks significant tidal features. We quantify the photometric properties of these structures, and discuss their origins in the context of each galaxy's environment and kinematics within the Virgo cluster.

Subject headings: galaxies: clusters: individual (Virgo) — galaxies: individual (M49, M84, M86, M87, M89)
\end{abstract}

\section{INTRODUCTION}

In a universe where structure grows hierarchically, the assembly of galaxies and galaxy clusters is characterized by an ongoing process of merging and accretion. These processes leave behind signatures which can be used to learn about the dynamical history of galaxies and clusters. On galaxy scales, structural asymmetries (e.g., Zaritsky \& Rix 1997; Conselice 2006; Coziol \& PlauchuFrayn 2007) and diffuse tidal streams and shells (Malin \& Carter 1980; Schweizer 1982, Tal et al. 2009) in field galaxies point towards recent accretion events. For galaxy clusters, X-ray substructure (West, Jones, \& Forman 1995; Mathiesen, Evrard, \& Mohr 1999), kinematic and spatial asymmetries in galaxy distribution (Dressler \& Shectman 1988; Knebe \& Müller 2000; Oegerle \& Hill 2001), and structure in the diffuse intracluster light (Feldmeier et al. 2004; Mihos et al. 2005, Rudick, Mihos, \& McBride 2006) are all signatures of dynamically young events.

In field galaxies, the tidal debris formed during galaxy interactions typically remains loosely bound to the galaxy (or merger remnant), forming tidal tails, loops, and shells that spatially mix over many Gyrs (Hernquist \& Spergel 1992; Hibbard \& Mihos 1995; González-García \& Balcells 2005). This tidal material is typically found at very low surface brightnesses $\left(\mu_{\mathrm{V}}>26.5 \mathrm{mag} / \operatorname{arcsec}^{2}\right)$, such that very deep imaging is needed to detect it. Such features have been used to reconstruct the dynamical history of nearby galaxies, or determine the role mergers

sjanowie@astro.indiana.edu,

craig.rudick@case.edu, heather@vegemite.case.edu

1 Department of Astronomy, Case Western Reserve University, Cleveland, OH 44106

2 Department of Astronomy, Indiana University, Bloomington, IN 47405

${ }^{3}$ Department of Physics and Astronomy, Youngstown State University, Youngstown, OH 44555 have played in the assembly of specific galaxies. For example, the tight interleaved shells seen in some elliptical galaxies are indicative of a radial merger with a lower mass companion (Quinn 1984), while long, luminous tidal tails are the hallmark of major mergers (Toomre \& Toomre 1972). Fainter tidal loops or streams are more ambiguous, and may come either from low mass accretion events (Bullock \& Johnston 2005) or the delayed fallback of tidal tails from a major merger (Hernquist \& Spergel 1992, Hibbard \& Mihos 1995). The variety of tidal morphologies thus provide an important archaeological tool for understanding the formation and growth of galaxies in the local universe.

For galaxies within clusters, the picture is more complicated. The lifetime of diffuse tidal structure depends on two competing processes: galaxy-galaxy and galaxycluster interactions which produce tidal debris, and the dynamical heating and mixing of tidal debris in the cluster environment as it is incorporated into the clusterwide ICL component (Rudick et al. 2006, 2009). The long-lived shells and loops seen in many field ellipticals may be absent in cluster ellipticals due to rapid tidal stripping of this loosely bound material by the cluster potential (Mihos 2004). Alternatively, the presence of such structure in a cluster elliptical could indicate that the galaxy is only now being accreted into the cluster, so that cluster processes have not yet had an opportunity to strip its diffuse tidal structure. While the variety of galaxy- and cluster-scale processes simultaneously at work during cluster assembly make it difficult to unambiguously extract the dynamical information held in tidal debris, it is clear that the information content in these features is rich, and provides important constraints on the dynamical history of these galaxies.

With these factors in mind, it is interesting to study the diffuse light around elliptical galaxies in a cluster environment. As part of our ongoing survey for ICL 
in the Virgo cluster (Mihos et al. 2005), we have obtained deep, wide-field imaging of the Virgo ellipticals M87 (NGC 4486), M86 (NGC 4406), M84 (NGC 4374), M89 (NGC 4552), and M49 (NGC 4472). These galaxies all occupy different environments within the cluster (see, e.g., Binggeli 1999 for a review of the structure of the Virgo Cluster). As the central dominant elliptical in the Virgo cluster, M87 lives near the center of the cluster potential well (as defined by the X-ray emission; Bohringer et al. 1994), and represents the center of the most massive subgroup of galaxies in the Virgo Cluster. M86 and M84 lie $\sim 1.3^{\circ}(370 \mathrm{kpc})^{4}$ to the northwest of M87; they are separated from each other by $17^{\prime}(78 \mathrm{kpc})$ in projection, close enough that, at faint surface brightnesses, their extended halos appear to merge together into a common envelope of light (Mihos et al. 2005). This is likely a projection effect, however, as distance estimates from surface brightness fluctuations place M84 about 1 Mpc behind M86 (Mei et al. 2007). Indeed, from a combined optical and X-ray analysis of the cluster, Schindler et al. (1999) suggest that M86 sits at the center of its own subcluster of galaxies merging with the main body of the cluster. M89 lies $\sim 1.2^{\circ}(335 \mathrm{kpc})$ to the East of M87 and is the least luminous of our selected elliptical galaxies. Finally, lying $4.4^{\circ}$ to the south of M87, M49 is the brightest elliptical in Virgo, and defines the center of another distinct Virgo subgroup (cluster B) which is dominated by spiral galaxies (Binggeli, Tammann, \& Sandage 1987). The different dynamical environments these ellipticals find themselves in is likely to translate to differences in the structure of their extended luminous halos.

In this work, we study the diffuse outer halos of these ellipticals, searching for tidal structures that may trace the dynamical histories of these galaxies. We use our deep imaging to fit and subtract a smooth elliptical fit to each galaxy's light profile, and identify tidal features in the residual images (for a similar approach, see, e.g., Canalizo et al. 2007). We then measure the total luminosity and peak surface brightness of each of the cataloged features. We describe the observational dataset and analysis techniques in $\S 2$, and detail the results for each galaxy in $\S 3$. Finally, we end with a discussion of these features in the more general context of the hierarchical assembly of galaxies and galaxy clusters in $\S 4$.

\section{IMAGING DATA}

\subsection{Observations}

The imaging data presented here were taken as part of our ongoing survey for diffuse intracluster light in the Virgo cluster (see, e.g., Mihos et al. 2005, 2009; Rudick et al. 2010) using Case Western Reserve University's 0.6/0.9m Burrell Schmidt telescope located at Kitt Peak National Observatory. The data were taken over the course of three observing seasons in Spring 2004, 2005, and 2006. M86 and M84 were imaged in Spring 2004, M89 in Spring 2005, M49 in Spring 2006, and M87 in both Spring 2004 and 2005 (see Table 1). In all seasons, the data were taken under dark, photometric conditions at airmasses less than 1.5. The SITe 2048x4096 CCD im-

\footnotetext{
${ }^{4}$ In this work, we adopt a Virgo distance of $16 \mathrm{Mpc}$ (see, e.g., Harris et al. 1998; Ferrarese et al. 2000; Mei et al. 2007); at this distance, $1^{\prime \prime}$ subtends $77.6 \mathrm{pc}$.
}

TABLE 1

Observational Datasets

\begin{tabular}{cccc}
\hline \hline Galaxy & Year & $N_{\text {image }}$ & $N_{\text {sky }}$ \\
\hline M87 & 2004 & 72 & 127 \\
M87 & 2005 & 45 & 103 \\
M87 & (total) & 117 & - \\
M84/M86 & 2004 & 72 & 127 \\
M89 & 2005 & 45 & 103 \\
M49 & 2006 & 51 & 78
\end{tabular}

Note. - $N_{\text {images }}$ refers to the number of images used in constructing each mosaic (2004 and 2005 data combined in the case of the M87 mosaic), while $N_{s k y}$ refers to the number of blank sky images used in flat fielding.

ages a field of view of $0.75^{\circ} \times 1.5^{\circ}$ using $1.45^{\prime \prime}$ pixels, and we build a larger mosaic by dithering our observations by up to one degree per field. Individual exposures are $900 \mathrm{~s}$ long, and mosaics are made using 45-117 dithered exposures in each field. Observations are taken through the Washington M filter, which is similar to Johnson $\mathrm{V}$ but bluer by $\sim 300 \AA$ and cuts out the strong and variable sky emission line at $[\mathrm{O}$ I] $\lambda 5577 \AA$.

To construct accurate flat fields, we image blank sky fields (typically $80-120$ per season) at nearly the same hour angle and declination as Virgo observations. This observing pattern reduces the effect on the flat fields of any flexure in the telescope system. Each night sky image had a 900s exposure time, and the brightness of the night sky varied from 1100-1500 ADU in the images. IRAF's OBJMASK task was used to mask stars and galaxies on the blank sky images, after which an iterative process was applied to fit and remove planar gradients in the sky levels before median combining the skies to create a super sky flat (see Feldmeier et al. 2002 and Rudick et al. 2010 for complete details).

Once flattened, the images are then star-subtracted to remove the extended wings of bright stars from the data. To construct the stellar point spread function, we start by using short exposures of moderately bright stars to define the small-scale PSF $\left(r<30^{\prime \prime}\right)$. At larger scales $\left(r>30^{\prime \prime}\right)$ we use 900 s exposures of $\alpha$ Leo to construct the PSF out to $r \sim 0.4^{\circ}$. The resulting large-scale $\left(r>10^{\prime \prime}\right)$ PSFs are shown in Figure1. Between the 2004 and 2005 seasons, the primary mirror was realuminized and the interior of the telescope tube was flocked with black velvet to reduce scattered light. These improvements reduced the brightness of stellar wings by about $2.5 \mathrm{mag} / \mathrm{arcsec}^{2}$ at large radius $\left(r \sim 20^{\prime}\right)$. With the reconstructed PSF, we then mask stars out to the radius where their scaled PSF falls below 3 ADU $\left(\mu_{\mathrm{v}} \approx 28\right)$ and then subtract the extended wings out to $0.5 \mathrm{ADU}\left(\mu_{\mathrm{V}} \approx 30\right)$. For the 2004 data reduction, star subtraction was done on the original images before sky subtraction and registration; for the 2005 and 2006 datasets, star subtraction was done after sky subtraction and registration. For the M87 dataset, which encompasses data taken both in 2004 and 2005, we have re-reduced the 2004 images for consistency; however, this test showed that differences between the two methods are small as long as sufficient area is available far away from bright stars to set the sky background.

Sky subtraction is problematic because the size of the Virgo Cluster is much bigger than the imaging field of 


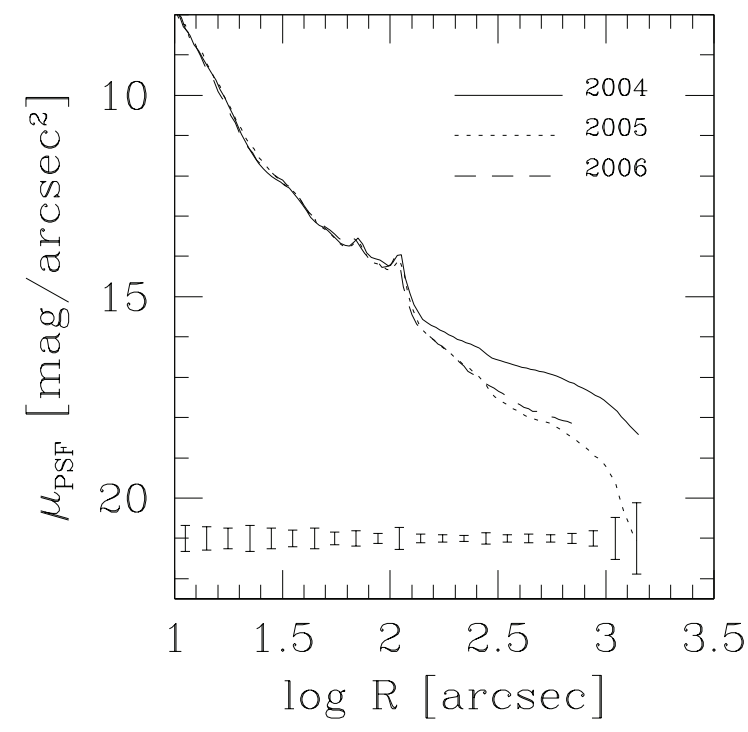

FIG. 1. - The stellar point spread function measured in the different datasets. Surface brightness is measured relative to the central surface brightness of the stellar profile (i.e., $\mu_{P S F r=0} \equiv 0$.) Realuminizing of the telescope mirror and flocking of the telescope tube between the 2004 and 2005 seasons resulted in a significant suppression of the stellar wings at large radius. The double peak near $\log \mathrm{R}=2$ is due to internal reflections within the Schmidt corrector. Errorbars along the bottom of the panel show characteristic uncertainties.

view. First, we mask the images of all sources brighter than $2.5 \sigma$ above sky, then spatially rebin the images by calculating the mode of the remaining unmasked pixels in 32x32 pixel "superpixels." Regions of images far away from bright stars and galaxies are then selected in which to measure a preliminary sky value to subtract. At this point, the binned images are registered and an iterative plane fitting process is employed to fit residual sky planes to the binned images, constrained to minimize frame-toframe variations in spatially-overlapping regions. The resulting edge-to-edge sky gradients which are removed from the images are typically about $5 \mathrm{ADU}$, or $<0.5 \%$ of the absolute sky level (1100-1500 ADU, or $\mu_{\mathrm{V}}=21.6$ 21.3). Once sky subtraction is complete, we register and median combine the individual images to create the final mosaic.

We quantify photometric uncertainty on two scales: small scale uncertainty due to read noise, photon noise, and small-scale flatfielding errors, and large scale uncertainties due to uncertainty in large scale flat fielding and sky subtraction. Small-scale uncertainties can be "root$\mathrm{N}$ reduced" by combining many individual images together into the final mosaic, and also by spatially binning the mosaic. Large-scale uncertainties do not reduce so easily, and we characterize them as a floor to our uncertainty, as described below. We refer the reader to Morrison et al. (1997), Feldmeier et al. (2002), Mihos et al. (2009), and Rudick et al. (2010) for more details of our error model as applied to the deep surface photometry.

For the CCD used in these observations, the gain was $2 \mathrm{e}^{-} / \mathrm{ADU}$ and readnoise was $12 \mathrm{e}^{-}$or 6 ADU. The typical sky background was 1250 ADU, which produces photon noise of 24.5 ADU per pixel. Photon noise in the blank skies used to construct the flat field will also result in small-scale flatfielding uncertainty (which will be mediated significantly by our dithering of object images). The resulting small-scale flat fielding error is 2.7 ADU (see Mihos et al. 2008). These errors add in quadrature to give an uncertainty of $25 \mathrm{ADU} /$ pixel in a single Virgo image. When median combining $N$ individual images together, the noise goes down as $1.22 \sqrt{N}$; for a typical dataset analyzed here, $N \approx 30$, giving a per pixel noise in the final mosaic of 0.6 ADU. Furthermore, when examining residual images, we spatially rebin the images in blocks of 9x9 pixels; these rebinned images then have a per pixel uncertainty of $<0.1 \mathrm{ADU}$ due to these smallscale effects.

Over larger scales, however, the dominant uncertainty lies in the large-scale flat fielding and sky subtraction. Flat fielding errors can come from a variety of imperfections in the blank sky images used to construct the flat, such as spatial variations in the sky brightness, the unsubtracted extended wings of bright stars, and diffuse low surface brightness structures such as galactic cirrus. Night sky variations in the object images also lead to uncertainty in the sky subtraction, as does the lack of substantial areas of pure sky in which to define the sky levels. Variability in the night sky happens both randomly and systematically (with, for example, hour angle, time of night, or airmass). To quantify these combined uncertainties, we measure the residual flux in 100x100 pixel boxes in blank sky regions in the flattened, skysubtracted mosaic. These boxes show a mean residual sky background of $0.1+/-0.5 \mathrm{ADU}$, and so we adopt a conservative $1 \mathrm{ADU}$ uncertainty as the floor to our error model. Given our photometric solution, 1 ADU corresponds to a surface brightness of $\mu_{\mathrm{V}}=29.2$.

\subsection{Ellipse Fitting and Model Subtraction}

Our approach to removing the smooth light profile from these galaxies involves fitting nested elliptical annuli over the galaxy's entire extent, and then subtracting the best-fit model from the image. As this fitting process is affected by all of the light in each isophote, it is imperative to remove any extra sources of non-galaxy light, lest they be interpreted as parts of the galaxy. The star mask and subtraction from the image reduction eliminates most of the light from the stars in each field, but other sources still remain. We have masked, by hand, all light in each image from nearby galaxies as well as the bright wings, diffraction spikes, and column bleeds around bright stars. To mask all small-scale sources of light, we use an automated source detection algorithm on an image with large scale structures removed. The large-scale structure is removed by smoothing the original image with a ring-median annulus of inner and outer radii of $4^{\prime \prime}$ and $12^{\prime \prime}$ (300pc and 900pc), and subtracting it from the original image. IRAF's OBJMASKS task is used to identify all sources $2 \sigma$ above sky which cover at least 2 pixels. This mask is combined with the previous mask to obtain our total mask. However, the galaxy centers are almost completely covered by this total mask. To obtain a reliable surface brightness profile fit, we unmask the central $2.5^{\prime}$ of each galaxy before fitting.

We use IRAF's ELLIPSE task to fit elliptical isophotes to each galaxy, which implements the iterative technique 

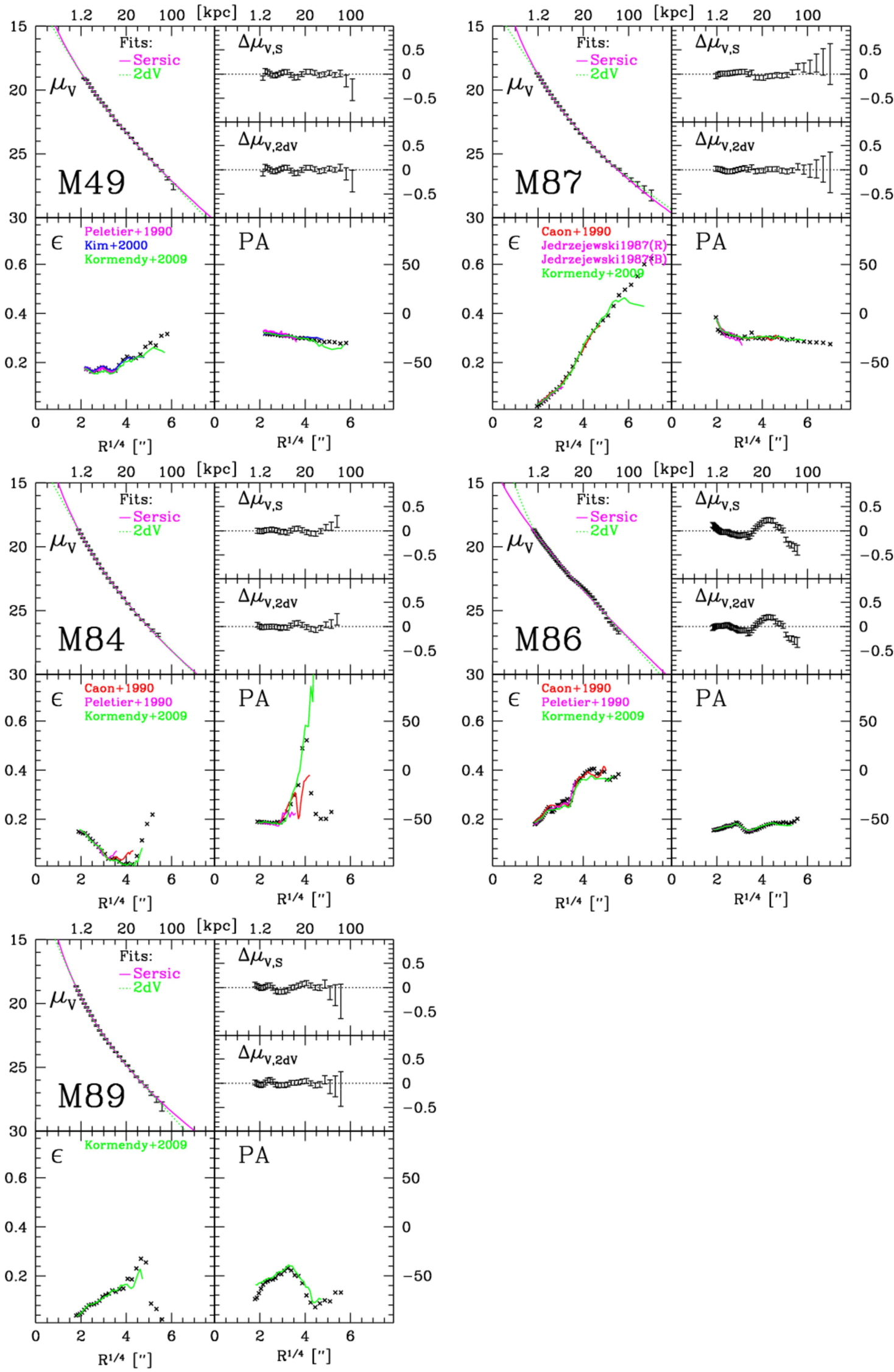

FIG. 2.- Isophotal fits for each of the five galaxies. The legend for each $4 \mathrm{x} 4$ panel is as follows: Upper left: Surface brightness profile, along with the best fit Sérsic (solid magenta line) and double deVaucouleurs (dotted green line) profiles. Upper right: Residuals from Sérsic $\left(\Delta \mu_{V, S}\right)$, and double deVaucouleurs $\left(\Delta \mu_{V, 2 d V}\right)$ surface brightness profile fits. Lower left: Ellipticity $(\epsilon)$ as a function of radius, with profiles from the literature over-plotted as noted. Lower right: Position angle (PA) as a function of radius, with profiles from the literature over-plotted as noted. 
described by Jedrzejewski (1987). At each step the geometric parameters of the elliptical isophote are varied until the residuals are minimized. For most of the galaxies we use a fixed center for the isophotes, which we determine by manually varying it until the inner $\sim 0.5^{\prime}$ shows no asymmetric residuals in the model-subtracted images. Within each isophote, the following parameters are computed: mean intensity, ellipticity $\left(1-R_{\mathrm{SMA}} / R_{\mathrm{SMB}}\right)$ and position angle (measured north through east). The fitting process only includes 1st and 2nd order Fourier terms (conveyed in the ellipticity and position angle) and does not fit the 3rd or 4th order terms. The 4th order $\left(A_{4}\right)$ component is a sign of disky or boxy isophotes (see Kormendy et al. 2009 and references therein), which will manifest in our subtracted images as a butterfly-like pattern of alternating light and dark wedges in the inner regions $\left(r \lesssim 0.5-1 r_{e}\right)$ of the galaxies. When later analyzing substructure, we only consider substructures outside of these features. We also avoid fitting the inner regions of the galaxies as they are often saturated and also our large pixel scale $\left(1.45^{\prime \prime} /\right.$ pixel $)$ is unable to capture the strong gradient around the center. In the low surface brightness outskirts of the galaxies, the robustness of the fit is determined by uncertainties in the sky subtraction. We continue fitting isophotes until we reach an isophotal surface brightness which is indistinguishable from sky. The estimated sky uncertainty in our images is $\sim 1 \mathrm{ADU}$; to determine the effects that this has on our fits, we carry out the same fitting procedure on an image which has 1 ADU added to every pixel and on another with 1 ADU subtracted. These two extra fits are how we obtain the error bars which are displayed on the best fit profiles.

Finally, we use IRAF's BMODEL task to make a model galaxy from the best fit elliptical isophotes. We subtract this model galaxy from the initial image and examine the residuals. The residuals contain both bright and dark regions, where the best-fit model under- and over-subtracts the light, respectively. Initially, more artifacts were evident in these residuals, around the edges of our hand mask, but we have iteratively adjusted our mask until all of the light coming from the other stars and galaxies is masked. We tried running the object detecting algorithm again on these residuals to improve the total mask, but found that the improvements in the fit and subtraction were minimal. This final subtraction is median smoothed into $7^{\prime \prime} x 7^{\prime \prime}$ bins, to bring out faint structures.

\subsection{Surface Brightness Profile Fits}

As a consistency check, we also fit the extracted surface brightness profiles to both Sérsic and double-de Vaucouleur profiles, and compare to values in the literature; the fitted parameters and their $1 \sigma$ uncertainties are reported in Table 2. In the fitting process, we take a rather conservative error model, wherein each datapoint is assigned an uncertainty equal to the maximum of $0.05 \mathrm{mag} / \operatorname{arcsec}^{2}$ or $1 \mathrm{ADU}$, whichever is larger. The former value corresponds to uncertainty in the photometric zeropoint and isophotal fitting process at high surface brightness, while the latter value refers to sky subtraction uncertainty, which dominates at low surface brightness. This error model leads to reduced $\chi^{2}$ values for the fits which are less than one, suggesting that this error model is a conservative one, overestimating the uncertainties in the fit. Since we are mostly interested in demonstrating consistency with other values reported in the literature, we do not further refine the error model. We have verified, however, that reasonable changes in the adopted uncertainties do not lead to large systematic differences in the extracted profile parameters.

Kormendy et al. (2009; hereafter K09) have recently published an extensive set of surface photometry for Virgo ellipticals, with detailed Sérsic fits. Our Sérsic fits are in reasonably good agreement with those of K09, with the fitted parameters typically agreeing to within $1-2 \sigma$. Where there is noticeable disagreement, the discrepancy can generally be attributed to the different radial ranges being fit between the two studies; as shown in K09, the fitted parameters can be sensitive to this choice 5 In surface photometry of more distant clusters, Gonzalez et al. (2005) have proposed that brightest cluster galaxies (BCGs) are better fit by double-deVaucouleur (hereafter " $2 \mathrm{dV}$ ") profiles, and that the properties of the outer profile are indicative of a diffuse intracluster component. Alternatively, Seigar et al. (2007) argue that Sérsic + exponential profiles are better able to fit the extended light from cluster $\mathrm{cD}$ galaxies. Our sample, of course, is not a sample of BCGs, but rather a sample of luminous ellipticals within an individual cluster, only one of which can be a BCG. Nonetheless, for comparison with these other studies, we fit our profiles to both Sérsic and doubledeVaucouleur models. In most cases, we find either fit acceptable, and therefore do not attempt fits which have additional fitting parameters. While the evidence for $2 \mathrm{dV}$ profiles as a superior function over single Sérsic is marginal at best in our dataset, we do calculate the relative amount of light in the inner and outer components of the $2 \mathrm{dV}$, for comparison with the results of Gonzalez et al. (2005).

\subsection{Photometric Measures of Substructure}

We employ a few methods to quantify the nature of the features visible in the residual images of the model subtraction. First, we identify individual features located outside the central regions $\left(r \lesssim 0.5-1 r_{e}\right)$ of the galaxies where higher order Fourier terms usually dominate the residual image and away from any other galaxies in the frame. To ensure that the features we identify are not artifacts of the subtraction process, we verify in all cases that they are present (although much harder to see) in the unsubtracted images as well. Once confirmed, we calculate the luminosity of these discrete features in polygonal apertures containing the features, and using adjoining regions to determine the local background for accurate background subtraction. The subtraction of a locally-defined background from the luminosity of each feature ensures that small-scale background uncertainty due to spatial variation of the subtracted galaxy model is minimized. Even so, the background subtraction remains the biggest source of uncertainty in measuring the flux in each region. We quantify this error by making small adjustments to the background regions to quantify the resulting background flux (see Rudick et al. 2010 for more details). We find this variation to be $\sim \pm 0.25$

\footnotetext{
5 We note that our quoted uncertainties in Table are standard $1 \sigma$ uncertainties, but direct the reader towards the more complete discussion of parameter uncertainties in Sérsic fits in K09.
} 
TABLE 2

Analytic Surface Brightness Profile Fits

\begin{tabular}{|c|c|c|c|c|c|c|c|c|c|c|}
\hline \multirow[b]{2}{*}{ Galaxy } & \multicolumn{4}{|c|}{ Sérsic fit } & \multirow[b]{2}{*}{ Galaxy } & \multicolumn{5}{|c|}{ Double deVaucouleur fit } \\
\hline & $n$ & $\begin{array}{c}r_{e} \\
{[\operatorname{arcsec}]}\end{array}$ & $\begin{array}{c}\mu_{e} \\
{\left[\mathrm{mag} / \operatorname{arcsec}^{2}\right]}\end{array}$ & $\chi^{2}$ & & $\begin{array}{l}r_{e}(\text { in }) \\
{[\operatorname{arcsec}]}\end{array}$ & $\begin{array}{c}\mu_{e}(\mathrm{in}) \\
{\left[\mathrm{mag} / \operatorname{arcsec}^{2}\right]}\end{array}$ & $\begin{array}{c}r_{e} \text { (out) } \\
{[\operatorname{arcsec}]}\end{array}$ & $\begin{array}{c}\mu_{e}(\text { out }) \\
{\left[\mathrm{mag} / \operatorname{arcsec}^{2}\right]}\end{array}$ & $\chi^{2}$ \\
\hline M49 & $6.90(0.46)$ & $311(20)$ & $23.8(0.29)$ & 0.80 & M49 & $52.4(20.2)$ & $21.2(0.5)$ & $423(86)$ & $24.5(0.6)$ & 0.75 \\
\hline M87 & $11.0(0.9)$ & $666(101)$ & $25.6(0.7)$ & 0.91 & M87 & $81.5(8.3)$ & $21.7(0.1)$ & $1753(440)$ & $27.5(0.5)$ & 0.26 \\
\hline M86 & $5.18(0.17)$ & $372(17)$ & $24.2(0.15)$ & 6.1 & M86 & $6.1(2.7)$ & $18.4(3.2)$ & $335(10)$ & $23.9(0.1)$ & 4.3 \\
\hline M84 & $9.75(0.85)$ & $204(18)$ & $23.8(0.5)$ & 0.55 & M84 & $40.4(6.9)$ & $20.9(0.25)$ & $501(128)$ & $25.9(0.6)$ & 0.61 \\
\hline M89 & $14.6(1.9)$ & $321(69)$ & $25.3(2.2)$ & 1.1 & M89 & $15.9(2.6)$ & $19.8(0.3)$ & $324(39)$ & $25.3(0.3)$ & 0.51 \\
\hline
\end{tabular}

Note. - Uncertainties in the fitted parameters are given in parentheses.

ADU, which we propagate to uncertainties in the derived luminosities.

As an independent check of the robustness of the derived substructure luminosities, we also reduced a completely independent dataset imaging M49 in 2005, and compared it to the results for the 2006 M49 dataset described here. We find the derived luminosities of the features agree to $\sim 5-10 \%$.

In addition to calculating the total luminosity of each feature, we also compute the peak surface brightness. We quantify this by constructing a $7^{\prime \prime} \times 7^{\prime \prime}(0.5 \times 0.5 \mathrm{kpc})$ median smoothed, model subtracted image, and examining the pixel intensities in each feature. To avoid statistical fluctuations associated with identifying the brightest binned pixel, we define peak surface brightness to be the 90th percentile of the intensity distribution. A visual examination of the images shows this to be robust against contamination from unsubtracted background objects and stars.

\section{RESULTS FOR INDIVIDUAL GALAXIES}

\subsection{M49 (NGC 4472)}

We begin with brightest Virgo elliptical, M49, located in the Virgo Southern Extension, four degrees south of M87. The final mask for M49 includes all of the bright stars in the field, as well as nearby faint galaxies (e.g. NGC4492, NGC4488). We fit the isophotal model using IRAF's ELLIPSE task, as described in $\S 2.2$, using fixed center (at $\alpha=12: 29: 46.8, \delta=+8: 00: 01.8$ J2000). Our extracted surface brightness profile never reaches our limiting magnitude of $\mu_{\mathrm{V}}=29$; the fit stops converging past $R_{\mathrm{SMA}}=32.8^{\prime}\left(\mu_{\mathrm{V}}=27.5\right)$ where too much of the galaxy extends off the edges of our image. The best fit profiles for surface brightness, position angle, and ellipticity are shown in Figure 2. We compare our results to Peletier et al. (1990) with CCD surface photometry in $B R$ bands, Kim et al. (2000) who used a CCD in Washington $C T_{1}$ bands, and the composite $V$ band profile of K09. As can be see in Figure 2, the ellipticity and position angle profiles are in very good agreement between these studies.

The isophotal fits to Sérsic and $2 \mathrm{dV}$ profiles are shown in Figure 2 and Table 2 For the Sérsic fit our extracted parameters agree reasonably well (within $2 \sigma$ ) with those measured by K09. There is no significant difference in the quality of fit between the Sérsic and $2 \mathrm{dV}$ models; extracting a total luminosity for the galaxies from the fitted profiles yields $L_{V}=1.6$ and $1.5 \times 10^{11} L_{\odot}$ for the Sérsic and $2 \mathrm{dV}$ fits, respectively. In the $2 \mathrm{dV}$ fit, the outer component carries $50 \%$ of the total galaxy luminosity.
We subtract the extracted profile from the original image of M49 to yield our residual image, shown in Figure 3. Strikingly visible in the residual image is a complex system of diffuse shells. A large shell sits 19.5' (90 kpc) to the northwest (Region 1), while a double-edged shell (Region 3) sits on the opposite side of M49, 12.5' (60 kpc) to the southeast. There is also a complex inner structure (Region 2) $7^{\prime}(30 \mathrm{kpc})$ to the northwest of M49, which appears to be the overlapping of several discrete shells. We also see a plume south of M49 center (Region 4), which partially overlaps the SE shell (Region 5). Finally, we identify a faint plume to the northeast (Region $6)$, running through the galaxy VCC 1254, and perhaps connecting with the inner shell structure. The peak surface brightnesses and total luminosities of these features are given in Table 3 . While these shells were not seen in the recent Tal et al. (2009) study of tidal features around nearby ellipticals, it is likely due to the extended nature of the shells; the field of view of the Tal et al. images was too small to reach most of these outer features. We also note at even fainter surface brightnesses a hint of a plume running to the northeast of M49, but this (unmarked) plume is only marginally detected, and we do not photometer it.

We note in the context of these shells that if the isophotal model has spurious features in it (due to rapidly changing ellipticity and position angle in the derived ELLIPSE model), the subtraction process can imprint shelllike artifacts into the residual image. We have been very careful in the fitting process to avoid these types of features. The fitted isophotal parameters vary smoothly with radius, and we have checked that the best-fit model did not have any structure that would be imprinted on the residual image, and we have also verified that all these structures can be recovered in the unsubtracted images.

The S Plume (Regions $4 \& 5$ ) also lies very near the tidally disturbed dwarf irregular UGC 7636 (in our image, UGC 7636 itself lies under the circular mask immediately north of the S Plume). Patterson \& Thuan (1992) studied UGC 7636 using optical and 21-cm imaging, and found short optical tidal tails extending $\sim 1^{\prime}$ north and south of the dwarf, and evidence for ram pressure stripping of its neutral hydrogen gas. The linear, low surface brightness S Plume is aligned with the relatively high surface brightness tails identified by Patterson \& Thuan, and is likely an extension of the southern tidal tail to larger radius. The narrowness of the plume argues, however, that this interaction is not the same that gave rise 
TABLE 3

M49 Substructure

\begin{tabular}{cccc}
\hline \hline $\begin{array}{c}\text { Region } \\
\#\end{array}$ & Name & $\begin{array}{c}\mu_{V, \text { peak }} \\
{\left[\mathrm{mag} / \operatorname{arcsec}^{2}\right]}\end{array}$ & $\begin{array}{c}\mathrm{L}_{\text {tot, } V} \\
{\left[10^{8} L_{\odot}\right]}\end{array}$ \\
\hline 1 & NW Shell & 27.6 & $2.28(0.18)$ \\
2 & Inner Shells & 27.0 & $2.03(0.11)$ \\
3 & SE Shell & 27.4 & $1.57(0.13)$ \\
4 & Overlap of 3\&5 & 27.4 & $0.60(0.04)$ \\
5 & S Plume & 27.0 & $0.51(0.04)$ \\
6 & NE Plume & 27.7 & $0.30(0.04)$ \\
\cline { 2 - 4 } & Total & $7.29(0.25)$ \\
\hline \multirow{2}{*}{ Total }
\end{tabular}

to the much broader, more extended NW and SE Shells.

The interleaved structure of those shells, roughly along the major axis of the M49, is very reminiscent of simulations of the accretion and subsequent disruption of small satellite galaxies around larger ellipticals (Quinn 1984, Hernquist \& Quinn 1988, 1989). While major mergers can also leave behind shell-like structures (e.g., Hernquist \& Spergel 1992), these are generally not so aligned and interleaved the way the shells in M49 are. These shells are also extremely sharp: a radial cut along the NW Shell (Region 1) shows a sudden $2 \mathrm{mag} / \operatorname{arcsec}^{2}$ drop over $35^{\prime \prime}$ $(2.7 \mathrm{kpc})$, while the SE Shell (Region 3) shows a steep $1.5 \mathrm{mag} / \operatorname{arcsec}^{2}$ drop over $50^{\prime \prime}(3.8 \mathrm{kpc})$, while the innermost shell (Region 2) has a much more diffuse decline in radial intensity. The fact that the sharpness of the shell is correlated with its distance from the center of M49 is consistent with an accretion origin, where the inner shells have experienced several dynamical crossings, resulting in heightened orbital mixing and diffusion of the shell's sharpness. The sharpness of the outer shell argues that it has been relatively unperturbed during its lifetime; we return to this implication in $\S 4$.

\section{2. $M 87(N G C$ 4486)}

The second galaxy we study is M87, the giant elliptical at the heart of the Virgo Cluster. Its final mask includes all of the small galaxies nearby (notably NGC 4478,4476 ) and several diffraction spikes and saturation bleeds from bright stars. Our fit uses a fixed center (at $\alpha=12: 30: 49.4, \delta=+12: 23: 28.2$ J2000), and extends outward to $R_{\mathrm{SMA}} \sim 40^{\prime}(180 \mathrm{kpc})$, where the surface brightness drops below $\mu_{\mathrm{V}}=29 \mathrm{mag} / \mathrm{arcsec}^{2}$, our $1 \sigma$ photometric limit. For display purposes, our subtracted images show residuals from an extended fit which continues into this sky level, but our quantitative discussion and profile fitting are restricted to the part of the profile with $\mu_{\mathrm{V}}<29$. Figure 2 shows M87's surface brightness profile and other best fit isophote parameters.

Also plotted in Figure 2 with our ellipticity and position angle profiles are results from similar previous studies of M87. Jedrzejewski (1987) published surface photometry of M87 in both B and R, while Caon et al. (1990) studied M87 using a combination of B-band CCD imaging and wide-field Schmidt photographic plates. K09 also combine their own observations with surface photometry published in the literature to construct a composite surface brightness profile of M87 in the V-band. While our data extend to somewhat larger semi-major axis, there is, for the most part, good agreement in the geometric isophotal parameters in regions of overlap. At large radius $\left(R^{\frac{1}{4}}>5.5\right.$, or $\left.R>15^{\prime}\right)$ there is some divergence in the ellipticity, but this is in regions of very low surface brightness $\left(\mu_{\mathrm{V}}>27.5\right)$ and may be due to contamination from galactic cirrus to the southeast of M87 (see discussion below).

The isophotal fits to Sérsic and $2 \mathrm{dV}$ profiles are shown in Figure 2 and Table 2, For the Sérsic fit, our extracted parameters agree well with those determined by K09. We note that $2 \mathrm{dV}$ is a somewhat better fit to the profile than the Sérsic model; however, much of the discrepancy between the fits comes in the outer regions at very low surface brightness. The total luminosity of M87 is $L_{V}=1.1$ and $1.2 \times 10^{11} L_{\odot}$ for the Sérsic and $2 \mathrm{dV}$ fits, respectively, and in the $2 \mathrm{dV}$ model the outer component accounts for $50 \%$ of the total luminosity.

We subtract the smooth isophotal model for M87 from the original image and show the subtracted image below the original in Figure 3. A major source of uncertainty is immediately visible in this image - contamination by back-scattered Galactic light from the galactic cirrus (Sandage 1976; Witt et al. 2008; Rudick et al. 2010). Much of the residual structure seen to the southeast (lower left) of M87 correlates strongly with far infrared maps of Galactic dust (Schlegel et al. 1998; MivilleDeschênes \& Lagache 2005), suggesting much of what we are seeing is due to scattering from galactic dust. It is particularly troublesome that this emission connects smoothly with the broad excess of light to the southeast of M87 which Weil et al. (1997) attributed to tidal encounter; this feature may be nothing more than scattered light from galactic cirrus. Given the likelihood of contamination by dust, we avoid attributing any substructure in this region to true tidal features around M87 itself. We limit our analysis to regions to the northwest of M87 which are largely free of dust contamination.

Highlighted in Figure 3 are several extended features surrounding M87. There is a wide plume extending radially to the north of M87's center (Region 4), and also two narrow streams extending to the northwest (Regions $1+2$ and 3) ${ }^{6}$ These radial streams are bright enough that they are easily visible even in the unsubtracted deep image (Mihos et al. 2005). The nearby galaxy PGC 41098 (VCC1148) also has a small stream emanating from it (Region 5). There is a hint that this stream continues to the northeast, running through the radial stream, and connecting up with the N Plume (Region 4), but this is just at our surface brightness limit we do not consider it firmly enough detected to photometer. The luminosity and maximum surface brightness of the detected features are given in Table 4

To assess the authenticity of all these features, and avoid confusion with galactic cirrus, we have compared them to far infrared IRIS observations over the same area (Miville-Deschênes \& Lagache 2005). While the spatial resolution of the IRIS data is only $4.3^{\prime}$, we find no correlation with regions of suspected galactic dust contamination, and are confident that these features are true

6 Regions 1 and 2 make up a single stream. Region 1 refers to the portion of the stream which extends beyond M87's halo, while Region 2 refers to the portion inside the halo. We photometer the stream separately in these two pieces, so that appropriate backgrounds can be applied. 

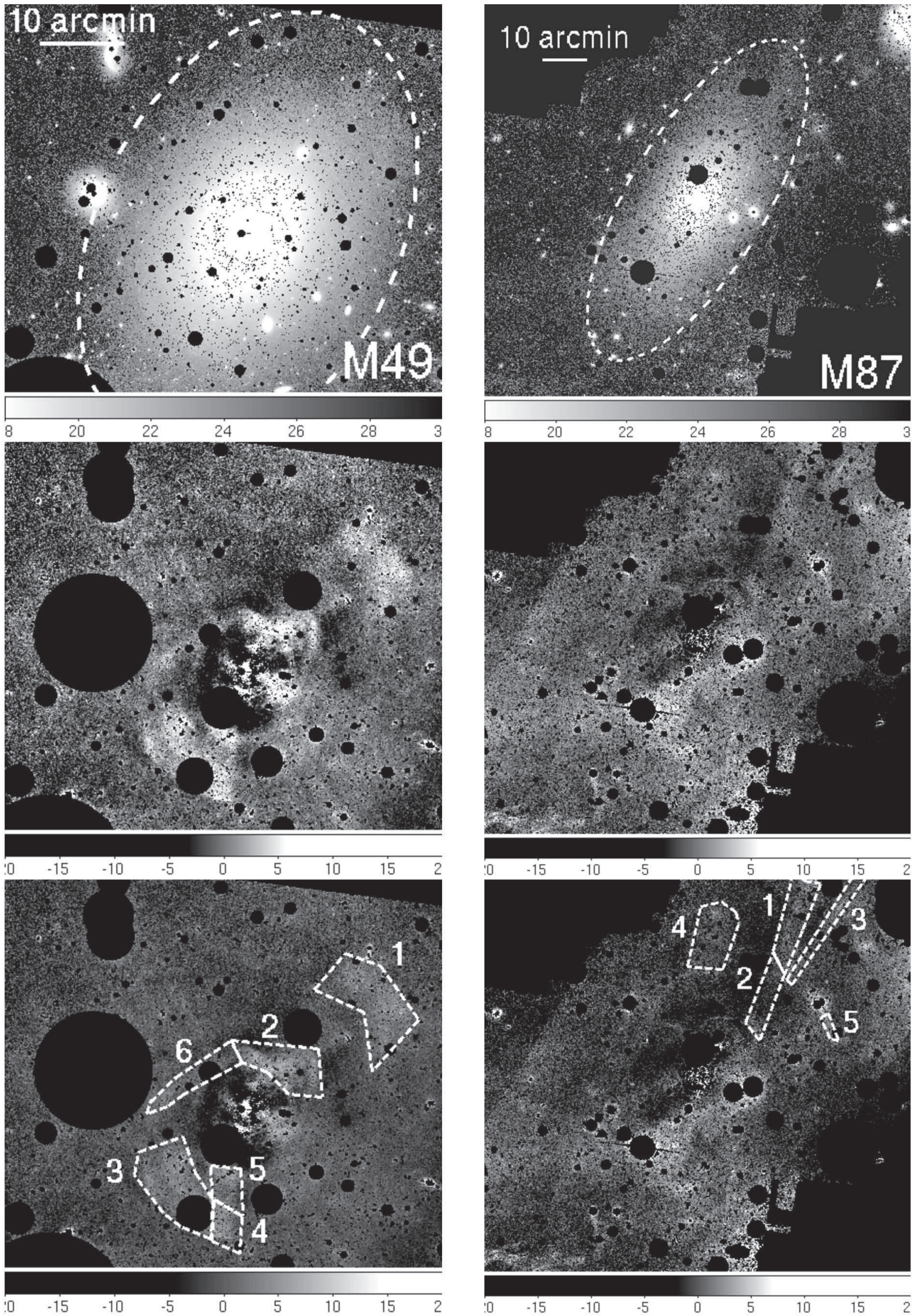

FIG. 3.- Deep imaging for M49 (left column) and M87 (right column). In each column, the top panel shows the original image, masked using the star mask from the data reduction. The greyscale legend shows surface brightness in $\mathrm{V} \mathrm{mag} / \mathrm{arcsec}^{2}$, and the overlaid ellipse outlines the $\mu_{\mathrm{V}}=29 \mathrm{mag} / \operatorname{arcsec}^{2}$ isophote. The middle panel shows the model subtracted residual image, median smoothed on $5 \times 5$ pixel $\left(7^{\prime \prime} \times 7^{\prime \prime}\right)$ boxes. In this panel, the mask shown is the final mask used in the fitting and subtraction process, and the greyscale legend shows brightness in ADU, where $1 \mathrm{ADU}$ corresponds to $\mu_{\mathrm{V}}=29.2 \mathrm{mag} / \mathrm{arcsec}^{2}$. The butterfly-like pattern near the center of M49 is caused by high order (A4) shape terms which are not captured in our ellipse fitting. We ignore these regions when searching for residual substructure. Similarly, we do not identify substructure in the southeastern (lower left) quadrant of the M87 image where there is significant contamination from galactic cirrus (see Rudick et al. 2010). The bottom panels show the same image as the middle panel, but with region identifier boxes overlaid. 
TABLE 4

M87 SubSTRUCTURE

\begin{tabular}{cccc}
\hline \hline $\begin{array}{c}\text { Region } \\
\#\end{array}$ & Name & $\begin{array}{c}\mu_{V, \text { peak }} \\
{\left[\mathrm{mag} / \mathrm{arcsec}^{2}\right]}\end{array}$ & $\begin{array}{c}\mathrm{L}_{\text {tot, } V} \\
{\left[10^{8} L_{\odot}\right]}\end{array}$ \\
\hline $1+2$ & NW Stream (N+S) & 27.8 & $2.35(0.28)$ \\
3 & WNW Stream & 28.3 & $0.53(0.18)$ \\
4 & N Plume & 28.1 & $1.41(0.31)$ \\
5 & W Arc & 28.2 & $0.13(0.02)$ \\
\cline { 2 - 4 } & Total & & $4.42(0.46)$ \\
\hline
\end{tabular}

Note. - Uncertainties on luminosities are given in parentheses.

stellar features around M87. Again, however, since we are explicitly avoiding the dust-contaminated regions to the southeast of M87, our catalog of features is likely underestimating the total structure around M87.

The long linear streams to the northwest of M87 are suggestive of small satellites falling in on radial orbits, or on more tangential orbits viewed along the orbital plane. The larger NW Stream (Regions 1+2) crosses the galaxy pair NGC 4458/61. These two galaxies have a velocity difference of $1300 \mathrm{~km} / \mathrm{s}$ and are not likely engaged in any slow mutual interaction that would draw out long tidal tails. It is possible, however, that the stripping of one of these galaxies as it orbits in the potential well of the cluster could have given rise to the NW Stream. The thinner WNW Stream (Region 3), projects across the dwarf galaxy VCC 1149, and again could be due to stripping of this galaxy as it orbits M87.

\section{3. $M 84 / M 86(N G C 4374 / 4406)$}

The surface brightness profiles of M84 and M86 overlap at large radii (where $\mu_{\mathrm{V}} \gtrsim 25$ ) and thus cannot be fit independently. We have employed an iterative process, by alternately fitting and subtracting both galaxies. Our fits show that M84 is better fit with a fixed center $(\alpha=12: 25: 03.8, \delta=+12: 53: 13.2 \mathrm{~J} 2000)$ and that M86's best fit isophotes have a center that drifts south east about 150 arcseconds from the initial center of ( $\alpha=12: 26: 11.8, \delta=+12: 56: 46.6$ J2000). The centroid drift is small at high surface brightness, but is more significant for the faint outer isophotes. At $\mu_{\mathrm{V}}=25$, the center has drifted less than $10^{\prime \prime}$, and the more significant drifting occurs fainter than $\mu_{\mathrm{V}}=27$.

We begin our iterative process by masking M86 and making an initial fit to M84, with limited radial extent. We subtract this M84 fit from the image, mask the residuals near M84's center, unmask M86, and make an initial fit to M86. We then subtract the M86 fit from the original image, again masking the inner residual, and make a new fit to M84, over a larger radial range. We continue this iterative process for 5 steps, until we have fit both galaxies out to $R_{\mathrm{SMA}}=34^{\prime}$ for M86 and $R_{\mathrm{SMA}}=24^{\prime}$ for M84. Because of the complexity of the fitting process, and the crowded nature of the field surrounding M84 and M86, our fits do not extend to the nominal limit of $\mu_{\mathrm{V}}=$ 29. As noted above, we find that as we reach $\mu_{\mathrm{V}}=27$, the centroid of the fit begins drifting significantly, at about the same point where the isophotes begin encompassing other galaxies in the field. We therefore take this brighter limit of $\mu_{\mathrm{V}}=27$ as the limit of our fitting process when extracting the analytic profile fits, and show this as our outermost isophote in Figure 4. The isophotal ELLIPSE fits for both M84 and M86 are shown in Figure 2.

We compare our surface brightness, ellipticity, and position angle profiles with those of Caon et al. (1990), Peletier et al. (1990), and K09 in $B, R$, and $V$ bands, respectively. For M84, we again find good agreement between those studies and ours, save for discrepancies in the position angle near $R_{\text {SMA }}^{1 / 4} \sim 4$. In this region, however, the ellipticity is so close to zero that the exact value of the position angle has little meaning. The Sérsic and $2 \mathrm{dV}$ fits for M84 (given in Table 2 yield a total luminosity of 7.2 and $6.5 \times 10^{10} L_{\odot}$ respectively, and in the $2 \mathrm{dV}$ fit the outer component carries $77 \%$ of the total luminosity.

For M86, the comparison between our profiles and those previously published is good throughout. In particular we note that the hump in the surface brightness profile of M86 near $R_{\mathrm{SMA}}^{1 / 4} \sim 4$ is also seen in the M86 profile of K09. This feature complicates the analytic fitting process (Table 2), yielding $\chi^{2}$ values significantly worse than for any other galaxy in our sample. Our Sérsic fit differs dramatically from that of K09, but as K09 shows, the range of radii chosen to fit the profile has a significant effect on the fit parameters. K09 do not fit past $R_{\mathrm{SMA}}^{1 / 4}=3.5$, and so their fit excludes the hump. If we limit our fit to a similar range, our fit parameters more closely match those of K09. The $2 \mathrm{dV}$ fit is similarly poor, consisting of a small high surface brightness inner component, and an outer component which contains $94 \%$ of the light. Given the actual shape of the profile, we do not consider this $2 \mathrm{dV}$ fit to be physically meaningful. The total luminosity of M86 is 9.3 and $9.2 \times 10^{10} L_{\odot}$ under the Sérsic and $2 \mathrm{dV}$ fits, respectively.

We subtract the combined M86 and M84 models from the original image, yielding the residual image shown in Figure 4. We note that in this case the mask displayed on the final residuals is a subset of the mask that is used in the fitting procedure. The bright galaxies south and east of M86 have all been aggressively masked in the analysis, but are displayed in this image for clarity. In the residual image, we immediately note the pinwheel-like fins centered on M86, which are indicators of the boxy isophotes noted by Peletier et al. (1990). These features are usually represented as azimuthal A4 Fourier components, beyond a pure elliptical model. Since we do not include these higher-order Fourier terms in our elliptical fits, these features show up as positive and negative residuals in the subtracted image. We restrict our discussion of the substructure around M86 to regions outside the area where these terms dominate the residual light profile.

Around M86, we find several small radial streams, including ones to the north (Region 1), south (Region 4), southeast (Region 5), and east (Region 6). These small features are very unlikely to be fitting artifacts, as they are coherent and consistent across many isophotes of the galaxies. We also find a few small plumes between M86 and M84 (Regions 2 and 3). We have verified that all these features can be seen in the unsubtracted image, and are therefore not artifacts of the subtraction process. It is tempting to link up features 1 and 4 , and features 2 and 6 , into continuous streams that cross the face of M86, as would be expected from tidal stripping of small orbiting satellites. The luminosity of these features is small, typically $2-3 \times 10^{7} L_{\odot}$ (Table 5 ). A dogleg stream of 

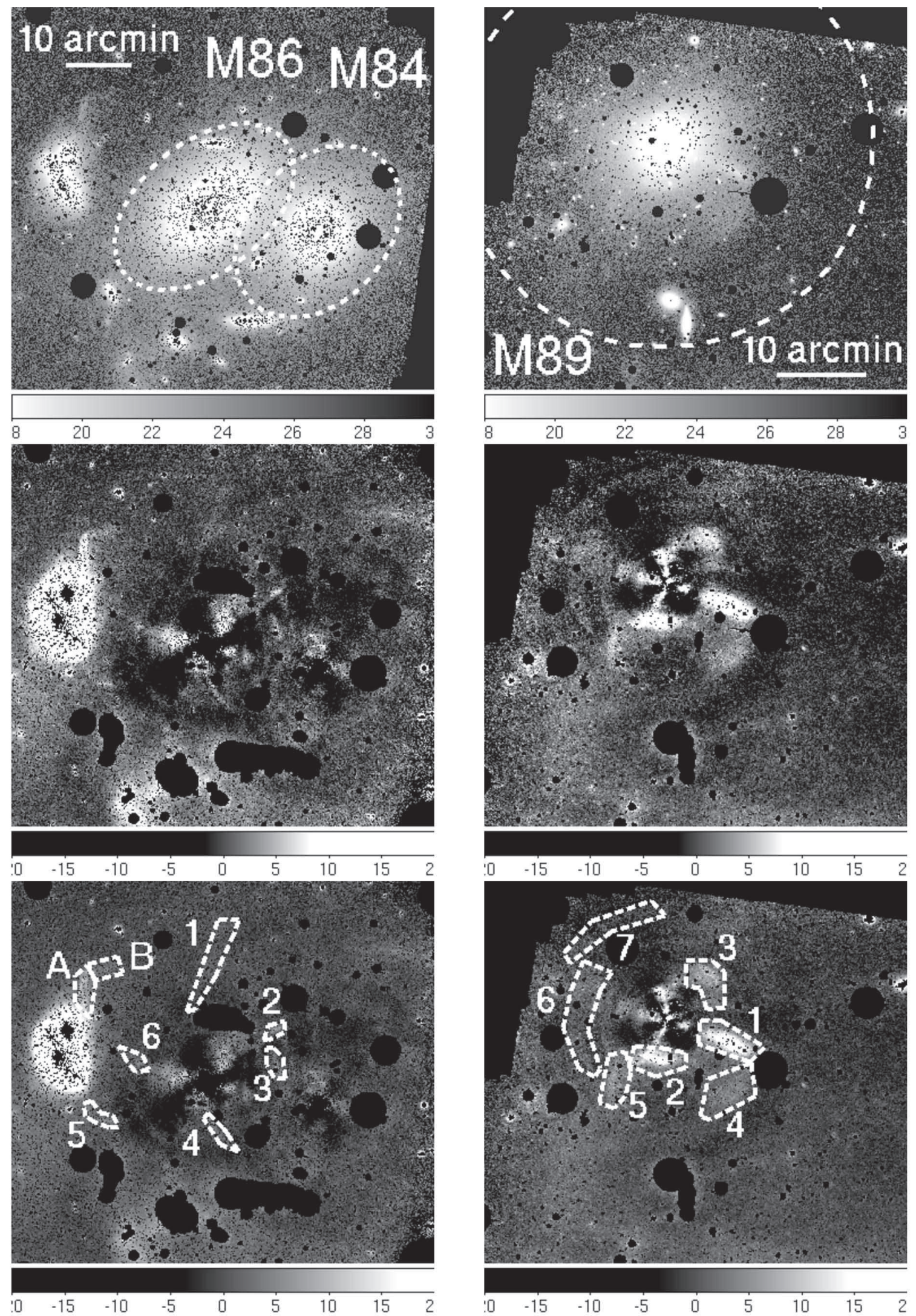

Fig. 4. - Deep imaging for M86/M84 (left column) and M89 (right column). In each column, the top panel shows the original image, masked using the star mask from the data reduction. The greyscale legend shows surface brightness in $\mathrm{V}$ mag/arcsec ${ }^{2}$, and the overlaid ellipse outlines the $\mu_{\mathrm{V}}=27 \mathrm{mag} / \operatorname{arcsec}^{2}$ (for M86/M84) and $\mu_{\mathrm{V}}=29 \mathrm{mag} / \mathrm{arcsec}^{2}$ (for M89) isophotes. The middle panel shows the model subtracted residual image, median smoothed on $5 \times 5$ pixel $\left(7^{\prime \prime} \times 7^{\prime \prime}\right)$ boxes. In this panel, the mask shown is the final mask used in the final photometering of the substructure, and the greyscale legend shows brightness in ADU, where $1 \mathrm{ADU}$ corresponds to $\mu_{\mathrm{V}}=29.2 \mathrm{mag} / \mathrm{arcsec}^{2}$. The butterfly-like pattern near the center of M49 is caused by high order (A4) shape terms which are not captured in our ELLIPSE fitting. We ignore these regions when searching for residual substructure. The bottom panels show the same image as the middle panel, but with region identifier boxes overlaid. 
light (Regions A and B) also extends from the nearby interacting pair of galaxies NGC 4435/8. This nature of this feature, first identified by Malin (1994), remains in doubt; optically it is bluer than other tidal streams in Virgo (Rudick et al. 2010), and multiwavelength observations suggest that it may in fact be a very unfortunate projection of galactic cirrus across the galaxy pair (Cortese et al. 2010).

We have also compared our deep optical image to the narrowband $\mathrm{H} \alpha$ image of Kenney et al. (2008), who found a very complex system of $\mathrm{H} \alpha$ filaments connecting M86 with NGC 4438. Kenney et al. proposed a collision between the ISM of the two galaxies as the source of the filaments. If these galaxies are in collision, we see no strong evidence of it in our imaging. We find no correlation between that $\mathrm{H} \alpha$ map and our deep imaging, and the few tidal features we see between the galaxies (Regions 5 and 6) are small and narrow, suggestive of small stripping of low velocity dispersion dwarf galaxies. However this does not rule out an encounter - the high relative velocities of M86 and NGC $4438(\Delta v=1379 \mathrm{~km} / \mathrm{s})$ would suppress the formation of strong tidal features, but still drive the strong response in the ISM/IGM giving rise to the ionized $\mathrm{H} \alpha$ filaments seen by Kenney et al. .

On smaller scales, Elmegreen et al. (2000) used optical imaging to identify a number of dust streamers 10-20 kpc from the center of M86, which they attributed to dust stripping from the dwarf elliptical VCC 882. Other evidence for dust stripping from galaxies orbiting M86 comes from far infrared ISOPHOT imaging (Stickel et al. 2003) which revealed a number of infrared sources with spectra consistent with cold dust emission lying within $35 \mathrm{kpc}$ from M86. These features are all close enough to M86 that they lie within the region where our ellipse subtraction is confused by the $A 4$ components in the galaxy profiles, and so we have not tried to identify features here (although we do see the optical dust lanes identified by Elmegreen et al. 2000). Nonetheless, the general inference that M86 is accreting and stripping a population of dwarf galaxies is consistent with the optical streamers we identify at larger radius.

As we did with M87, we have compared our residual image with the far infrared maps to guard against confusion with galactic cirrus. The infrared maps show significant dust contamination to the west of M84; some of this cirrus can be seen as the (unlabeled) diffuse feature seen in the upper right edge of our image. There is also infrared emission associated with the dogleg plume near NGC 4435/8, one of the arguments for its identification as galactic cirrus (Cortese et al. 2010). Other than these features, we see no clear correspondence between the infrared dust emission and the diffuse streamers we have identified near M84 and M86.

\section{4. $M 89$ (NGC 4552)}

The elliptical galaxy M89 has the lowest luminosity in our sample, and resides one degree east of M87. M89's final mask eliminates all of the bright stars and small galaxies in the field of view, and we also mask the bright shell to the south and the strong "jet" feature to the west, both identified first by Malin (1979). Since these features are obvious substructures distinct from the smooth galaxy light, we want to exclude them from contributing to the elliptical isophotal fit. We again use a fixed
TABLE 5

M84/M86 SubstruCTURE

\begin{tabular}{ccccc}
\hline \hline $\begin{array}{c}\text { Reg. } \\
\#\end{array}$ & Name & Galaxy & $\begin{array}{c}\mu_{V, \text { peak }} \\
{\left[\mathrm{mag} / \text { arcsec }^{2}\right]}\end{array}$ & $\begin{array}{c}\mathrm{L}_{\text {tot }, V} \\
{\left[10^{8} L_{\odot}\right]}\end{array}$ \\
\hline 1 & N Stream & M86 & 28.3 & $0.36(0.09)$ \\
2 & N Middle Plume & $?$ & 27.6 & $0.17(0.02)$ \\
3 & Middle Plume & $?$ & 27.7 & $0.19(0.03)$ \\
4 & S Stream & M86 & 28.1 & $0.18(0.02)$ \\
5 & ESE Plume & M86 & 27.8 & $0.18(0.03)$ \\
6 & E Stream & M86 & 28.2 & $0.19(0.02)$ \\
\cline { 2 - 5 } & Total & & M86 & $0.91-1.27(0.10)$ \\
& Total & & M84 & $0.00-0.36(0.04)$ \\
\hline
\end{tabular}

Note. - Uncertainties in the luminosities are given in parentheses. The total luminosities are given as ranges, depending on whether the luminosity of Regions 2 and 3 is given to M86 or M84.

center (of $\alpha=12: 35: 39.8, \delta=+12: 33: 23.2$ J2000) for our fit, and our best-fit surface brightness profile reaches our limiting magnitude of $\mu_{\mathrm{V}}=29$ at $R_{\mathrm{SMA}}=23^{\prime}$. Our best fit elliptical model is shown in Figure 2, where we also compare our surface brightness profile with that of K09, who combined original observations with published data to construct a composite $V$-band profile for M89. The comparison shows an excellent match in ellipticity and position angle.

Our best-fit Sérsic and $2 \mathrm{dV}$ fits to the luminosity profile are shown in Figure 2 and the parameters are given in Table 2 Our fitted Sérsic model has an extremely high index of $n=14.6$, similar to the value of $n=13.9$ found by Caon et al. (1993), but higher than the $n=9.2$ reported by K09. Again, however, the radial range of the fit is important; in a fit with a radial range that more closely matches ours, K09 derive a larger value of $n=13.75$ (see Figure 56 of K09). In our fits, the $2 \mathrm{dV}$ model yields a somewhat better fit than the Sérsic model, with the outer component contributing $68 \%$ of the total luminosity of $L_{V}=3.9 \times 10^{10} L_{\odot}$. The Sérsic gives a higher total luminosity of $L_{V}=4.9 \times 10^{10} L_{\odot}$.

Subtracting our isophotal model from the raw image yields the residual image shown in Figure 4. As in the case of M86, we see a strong signature of boxy A4 components in the inner parts of the galaxy, and restrict our study of substructure to regions at larger radius. A great deal of the area near M89 is covered in substructure. Easily visible are the "jet" feature to the west (Region 1) and the extremely bright shell to the south (Region 2). We also find plumes to the northwest (Region 3), southwest (Region 4), and southeast (Region 5) of M89. Fainter and further away are two shells: one $9.3^{\prime}(42 \mathrm{kpc})$ to the east (Region 6), and another $12.3^{\prime}$ (57 kpc) to the northeast (Region 7). As with the previous galaxies, we have confirmed that all these features are visible in the unsubtracted image as well. Photometric measurements were made of each of the detected features and compared with directly adjacent sky regions. Table 6 gives the photometric properties for each feature.

The variety of tidal structure around M89 argues for a complicated accretion history. The W Tail (Region 1) is peculiar in that it has a high, relatively constant surface brightness across its length, and may be a long tidal tail seen curving back on itself in projection. Indeed there is some hint of a plume extending to the east of M89 along the same axis as the $\mathrm{W}$ Tail, projecting across the 
TABLE 6

M89 Substructure

\begin{tabular}{|c|c|c|c|}
\hline $\begin{array}{c}\text { Region } \\
\#\end{array}$ & Name & $\begin{array}{c}\mu_{V, \text { peak }} \\
{\left[\mathrm{mag} / \operatorname{arcsec}^{2}\right]}\end{array}$ & $\begin{array}{c}\text { Ltot, } V \\
{\left[\begin{array}{cc}10^{8} & L_{\odot}\end{array}\right]}\end{array}$ \\
\hline 1 & W tail & 25.7 & $2.56(0.07)$ \\
\hline 2 & $\mathrm{~S}$ shell & 26.3 & $1.62(0.05)$ \\
\hline 3 & NW plume & 26.9 & $1.75(0.07)$ \\
\hline 4 & SW Plume & 27.8 & $1.09(0.12)$ \\
\hline 5 & SE Plume & 27.6 & $0.70(0.05)$ \\
\hline 6 & E Shell & 27.9 & $1.45(0.23)$ \\
\hline \multirow[t]{2}{*}{7} & NE Shell & 28.2 & $0.57(0.10)$ \\
\hline & Total & & $9.74(0.30)$ \\
\hline
\end{tabular}

Note. - Uncertainties in luminosities are given in parentheses.

outer shells - if real, this could be the extension of the $\mathrm{W}$ tail curving back across the face of the galaxy. Unlike the shell system of M49, M89's shells do not have the classic aligned and interleaved structure expected from phase wrapping from a small accretion event (e.g., Quinn 1984). Instead, the shells occur at a variety of position angles and radii, suggesting material that has come into the galaxy with a range of angular momenta. Such features could arise either from multiple accretions, or via a major merger of two disk galaxies, where tidal material can spatially wrap around the remnant without any coalignment of shells (e.g., Hernquist \& Spergel 1992; Hibbard \& Mihos 1995). The sharpness of the outer shells (Regions 6 and 7) also argues that, like M49, M89 must not have experienced significant tidal stripping from the Virgo cluster environment over the past Gyr or so, otherwise the shells would have been disturbed or disrupted.

\section{SUMMARY AND DISCUSSION}

In summary, we have used deep, wide-field surface photometry to study the extended envelopes of five luminous Virgo ellipticals. We use the IRAF ELLIPSE task to fit elliptical isophotes to the galaxies' surface brightness profiles. Analytic fits to these isophotal models compare well to fits published elsewhere in the literature. Subtracting the isophotal models from the images, we identify a variety of low surface brightness tidal features - streams, plumes, and shells - in the outer envelopes of these elliptical galaxies, which we have quantified in terms of their total luminosity and peak surface brightness. We find that M87 is characterized by an extended diffuse halo with broad plumes and radial streams, but no sharp tidal shells or loops. M86 shows a number of small, relatively high surface streams, while M84 shows no evidence for significant substructure beyond its smooth elliptical isophotes. In contrast, both M49 and M89 show complex (and in M49, hitherto undiscovered) systems of distinct shells and other tidal features.

The variety of structure we see in these Virgo ellipticals may well reflect differences in their history of accretion of smaller galaxies, their accretion history into the Virgo Cluster itself, or a combination of the two. To begin our discussion of environmental influences, we show in Figure 5 three orthogonal projections of the three dimensional positions of our sample galaxies in the Virgo cluster. We use the distances to each galaxy derived by Mei et al. (2007) using the surface brightness fluctuation technique. We adopt this dataset largely because it has consistently derived distances for all of our galaxies, with
TABLE 7

Galaxy Properties

\begin{tabular}{lccccc}
\hline \hline Galaxy & $\begin{array}{c}R_{p}{ }^{\mathrm{a}} \\
{[\mathrm{Mpc}]}\end{array}$ & $\begin{array}{c}r_{3 d^{\mathrm{b}}} \\
{[\mathrm{Mpc}]}\end{array}$ & $\begin{array}{c}v_{\text {rel }}{ }^{\mathrm{c}} \\
{[\mathrm{km} / \mathrm{s}]}\end{array}$ & $\begin{array}{c}L_{\text {sub }} \\
{\left[10^{8} L_{\odot}\right]}\end{array}$ & $\begin{array}{c}f_{\text {sub }} \\
{[\%]}\end{array}$ \\
\hline M49 & 1.23 & 1.32 & -67 & $7.29(0.25)$ & $0.47(0.02)$ \\
M87 & $\equiv 0$ & $\equiv 0$ & 243 & $4.42(0.46)$ & $0.39(0.04)$ \\
M86 & 0.35 & 0.54 & -1308 & $0.91-1.27$ & $0.10-0.14$ \\
M84 & 0.42 & 1.32 & -4 & $0.00-0.36$ & $0.00-0.05$ \\
M89 & 0.33 & 1.41 & -724 & $9.74(0.30)$ & $1.99(0.06)$
\end{tabular}

a Projected clustercentric distance, defined relative to M87

b Three dimensional clustercentric distance

c Velocity relative to cluster velocity of $+1064 \mathrm{~km} / \mathrm{s}$ (Binggeli et al. 1999)

very low internal errors. However, there are systematic uncertainties in the absolute distances; other techniques yield somewhat different results. Most notably, the position of M86 is quite uncertain - while the Mei et al. distances place M86 in the cluster core, in front of M84, distance determinations derived from planetary nebula put both M86 and M84 at the same distance, 1 Mpc behind M87 (Jacoby et al. 1990). Finally, Figure 5 also plots the line of sight velocity vectors of each galaxy, after subtracting out a mean Virgo velocity of $1064 \mathrm{~km} / \mathrm{s}$ (Binggeli 1999), and the estimated r200 (1.55 Mpc) for Virgo, determined by McLaughlin (1999).

Without transverse velocities for these galaxies, a full dynamical interpretation is impossible; nonetheless, we can glean useful information from this plot. As Virgo's central galaxy, it is no surprise that M87 has a low line of sight motion $\left(v_{\text {rel }}=243 \mathrm{~km} / \mathrm{s}\right)$ with respect to the cluster as a whole. Sitting $\sim 1 \mathrm{Mpc}$ behind the cluster core, M84's low velocity $(-4 \mathrm{~km} / \mathrm{s})$ suggests it is either near apocenter on a low angular momentum orbit - likely having passed near the cluster center a few Gyr ago - or moving on a more tangential orbit which keeps it out of the cluster center. As M49 is not projected onto the cluster core, its low velocity $(-67 \mathrm{~km} / \mathrm{s})$ places little constraint on its orbit. However, the presence of an X-ray bowshock to the north of M49 argues that the galaxy is falling into Virgo's hot intracluster medium from the south (Irwin \& Sarazin 1996). Only M86 and M89 have significant line of sight motion with respect to the cluster center $(-1308 \mathrm{~km} / \mathrm{s}$ and $-724 \mathrm{~km} / \mathrm{s}$, respectively). M86 is either moving at high speed through the core, or just about to enter it, depending on the adopted distance, while M89 either passed through the core $\sim 1-2$ Gyr ago (if it lacks significant transverse velocity) or is on a more tangential orbit that keeps it further from the core.

We first examine the environmental question by looking to see if the amount of diffuse substructure in the galaxies correlates with their distance from the center of Virgo (taken to be the position of M87). We quantify the amount of substructure in two ways: the total luminosity in the features $\left(L_{\mathrm{sub}}\right)$, and their fractional luminosity ( $f_{\text {sub }}$, measured with respect to the total galaxy light). In the latter case, the galaxy luminosities are calculated analytically from our Sérsic surface brightness fits. We then calculate each galaxy's distance from the center of the Virgo Cluster using a combination of their projected distance from M87 on the sky and their line-of-sight distance from Mei et al. (2007). These quantities are given 

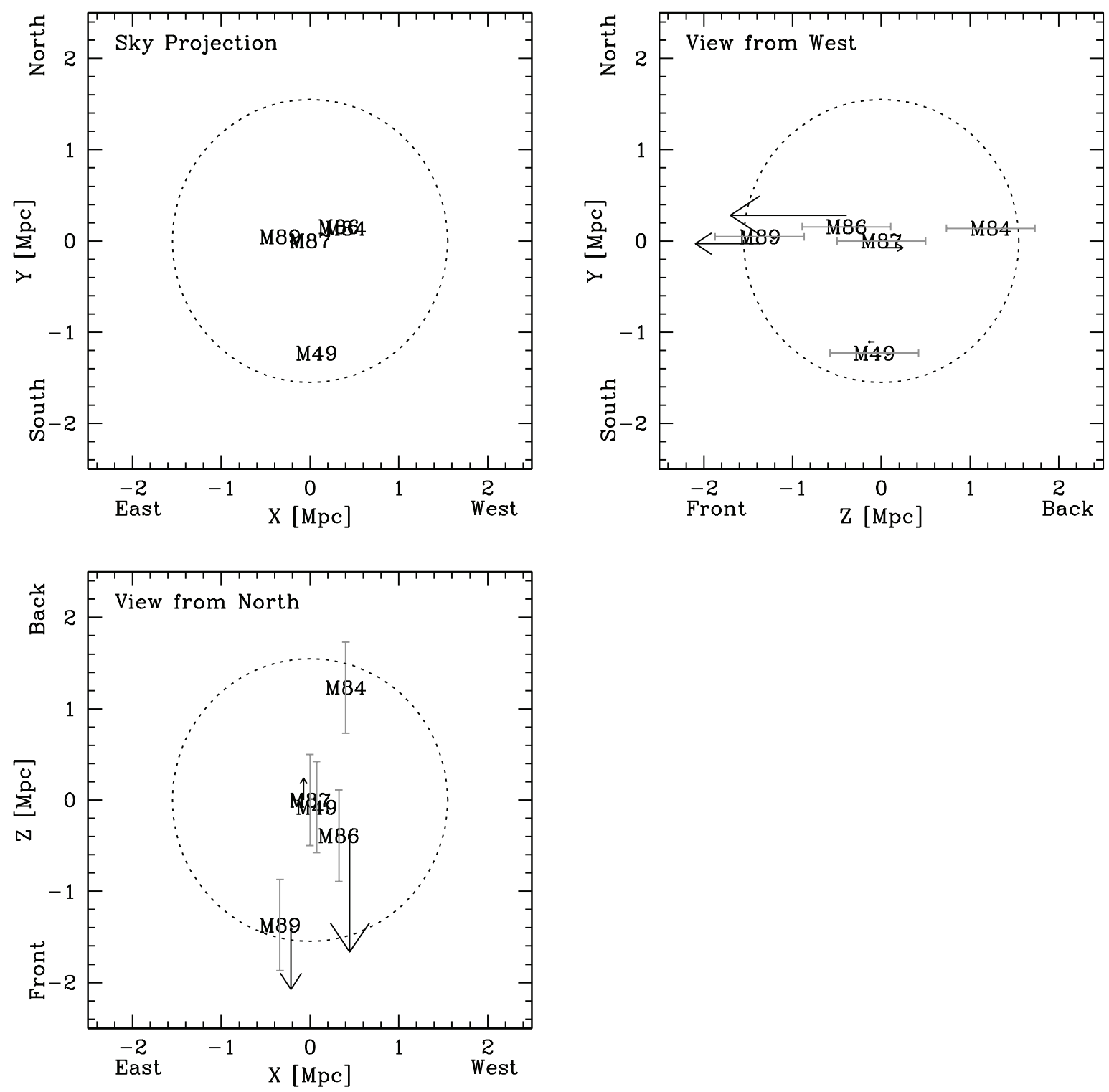

FIG. 5. - The three dimensional geometry of the Virgo cluster, using line of sight SBF distances from Mei et al. (2007). In each panel, the dotted line shows the virial radius $\left(r_{200}\right)$ for Virgo from McLaughlin (1999). Arrows show the line of sight velocity of each galaxy, with the length of the arrow equal to the distance traveled in 1 Gyr.

\section{in Table 7.}

For these five galaxies, we see no obvious correlation between the amount of substructure and cluster-centric distance, as shown in Figure 6. M87, M49, and M89 have significantly more substructure luminosity than the others, although in the case of M87 and M49, the substructures contribute much less to the total luminosity of the galaxies. Neither M84 nor M86 appear to have appreciable substructure, either in total or fractional luminosity. A model where substructure survival depends simply on cluster-centric distance is clearly too simplistic to explain the features seen in these Virgo ellipticals.

However, M87 is not simply deep in the cluster poten- tial, it lives at the center of the cluster. In this privileged position, it experiences a constant rain of smaller satellite galaxies falling in at high velocities, which will be tidally stripped and leave behind long streamers such as those seen extending to the NW of M87. Unlike the long-lived structures around isolated galaxies, streamers inside an active cluster environment are dispersed rapidly via interactions with other cluster members, typically within a few crossing times (Rudick et al. 2009). In this position, M87 has both high creation rates and high destruction rates for its diffuse substructure, so that the window of opportunity for observing cold streams around M87 will be short. 

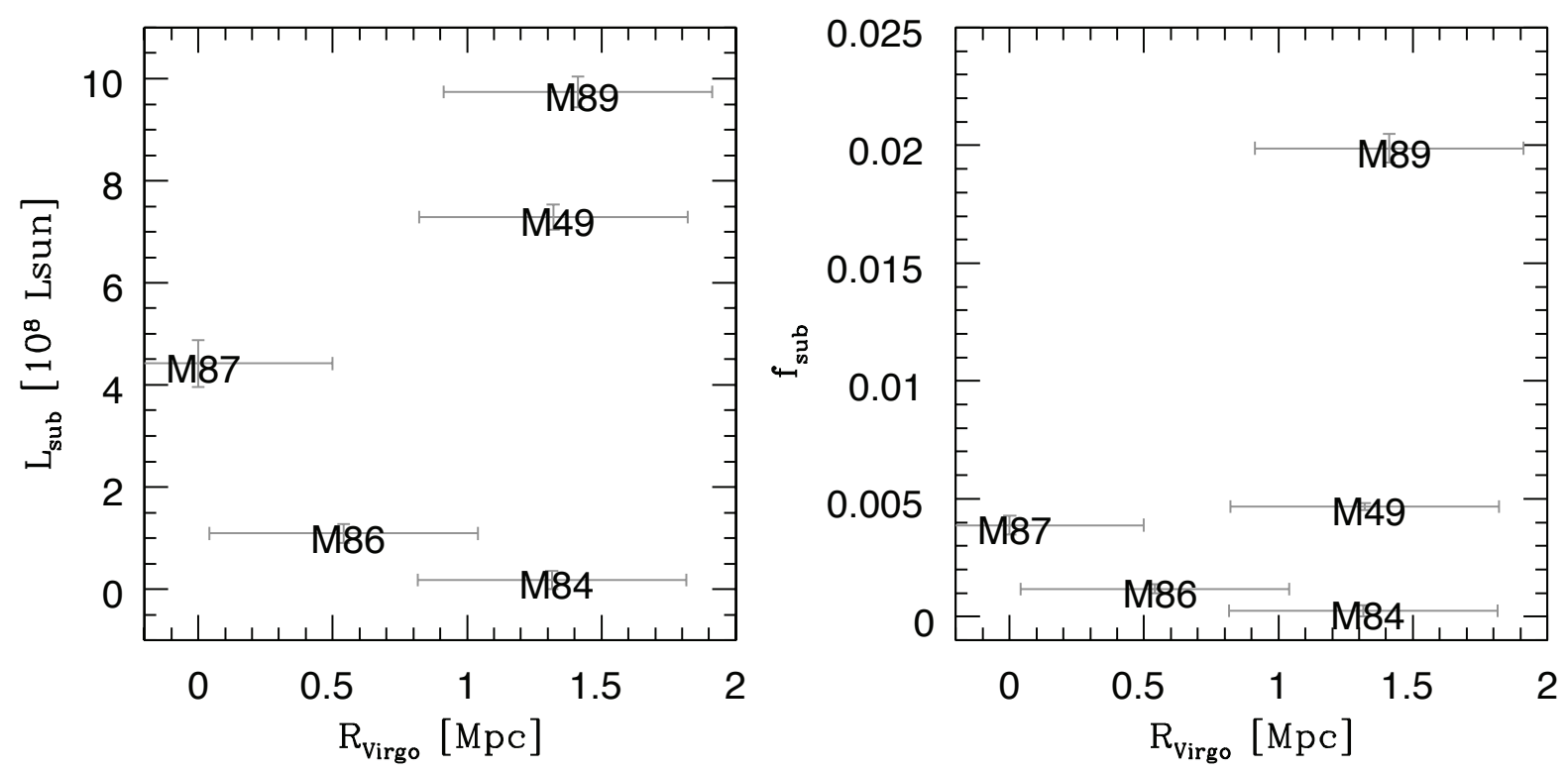

FIG. 6.- Substructure luminosity (left) and luminosity fraction (right) as a function of clustercentric distance.

While the amount of substructure does not seem to correlate with cluster-centric radius, there are interesting patterns in the morphological properties of the diffuse light in these ellipticals. M49 and M89 both have complex shell structures out to large radius, where the material would be loosely bound to the host galaxy. Any strong tidal forces that would occur during a galaxy's passage through the cluster would likely be sufficient to strip or at least significantly perturb the shells (see, e.g., Mihos 2004). The sharpness of the shells and the long dynamical timescales in the outskirts of these galaxies $(\sim 0.5$ Gyr $)$ argue that these galaxies have not passed through the dense Virgo Core in the recent past. In contrast, we see little evidence for sharp shell-like structures in M87, M86, or M84. Living at the center of Virgo, M87 experiences repeated encounters with cluster galaxies, and any dynamically delicate feature will quickly be destroyed. In the radial streams visible to the northwest of M87, we are likely seeing very recent stripping of galaxies falling in on radial orbits.

The nature of the streams observed in M87 and M86 is qualitatively different as well. M87's NW and WNW streams can be traced out to $\sim 1$ degree $(275 \mathrm{kpc})$ from the center of M87, while M86's streams are much smaller. The longest, the N Stream, extends 25' from M86, while the others are much smaller in length (only a few arcminutes in size) and closer to M86. Moving at such a high velocity near or through the cluster core, M86 simply may not be able to hang on to extended debris. The cluster tidal field may easily strip these streams, or they may lack coherency simply because of the motion of M86. A coherent stream at $300 \mathrm{kpc}$ would have an orbital period of a few Gyr, a timescale over which M86 would have moved a few Mpc through the cluster. The combination of tidal effects and M86's high speed motion through the cluster would make it difficult for M86 to retain highly extended streamers.

Substructure is not the full story of the diffuse light in Virgo ellipticals, of course. Because these features are likely to be short-lived in a dynamically complex environment like a galaxy cluster, they will mix into a more diffuse, extended envelope. Whether or not this envelope is a dynamically or structurally distinct entity from the galaxy itself is a subject of considerable debate. Gonzalez et al. (2005) argue that in clusters with clear BCGs, the intracluster light settles into a structurally distinct component from the galaxian light of the BCG, and model the two components using distinct $r^{1 / 4}$ profiles. They find that, in their sample of BCGs, the outer profile typically contains $\sim 80-90 \%$ of the total luminosity, and has an effective radius 10-40 times larger than the inner component. If we consider M87 in this way, we find a smaller fraction of light in the extended envelope (50\%) than typical in the Gonzalez et al. sample. However, Virgo is somewhat different from those BCG clusters, in that it has a number of comparably bright galaxies (M87 at the center; M86 projected $0.5^{\circ}$ away; and M49 $2^{\circ}$ to the south). With multiple bright galaxies and both spatial and kinematic substructure, Virgo may represent a dynamically less-evolved progenitor to the Gonzalez et al. clusters, in which case the lower fraction of light in the envelope may be a signature of an still-developing ICL (e.g., Rudick et al. 2006). This inference echoes that of K09, who argue based on the systematics of M87's Sérsic fit that the galaxy is at best only a weak $\mathrm{cD}$ galaxy.

In this context, it is also interesting to look at M49. M49 is the dominant galaxy in the Virgo Southern Extension (VSE), and somewhat more luminous than M87. If clusters build through the hierarchical accretion of subclumps, the sub-clumps themselves may have their own diffuse light component, as suggested in the simulations by Rudick et al. (2006). If the VSE is an evolved group 
now being accreted into the Virgo cluster, it may have a structure similar to (albeit smaller than) that of the BCG clusters of Gonzalez et al. (2005). And indeed, the $2 \mathrm{dV}$ fit for M49 shows a higher fraction of light $(76 \%)$ in the outer component than does M87, but with a much smaller characteristic scale $\left(r_{e, \text { out }}(\mathrm{M} 49)=20 \%\right.$ $\left.r_{e, o u t}(\mathrm{M} 87)\right)$.

While these results are consistent with a scenario of a gradual, on-going buildup of ICL in Virgo, there are several important caveats. First, the $2 \mathrm{dV}$ profiles are not clearly superior to regular Sérsic fits, and the justification for dividing luminosity into an inner and outer component based on these fits is not strong. Even the choice of functional form for the division is the source of some debate - Seigar et al. (2007) argue that an inner Sérsic + outer exponential fit is a better description of the light profile for $\mathrm{cD}$ galaxies. However, given the fact that the additional free parameter of the $2 \mathrm{dV}$ fit over the Sérsic fit did not result in significantly better profile fits for our galaxies, we do not pursue these higher order fits here.

Given our results, we paint a plausible picture of the dynamics of the galaxies within the Virgo Cluster. Sitting at the center of the cluster, M87 experiences a rain of smaller galaxies which are being tidally stripped by the cluster potential, leading to the long diffuse streams seen to the NW of M87. Due to encounters with other galaxies in the cluster core, the lifetime of these streams is short and they mix away to continually build M87's extended envelope and Virgo's intracluster light. The combination of M86's high velocity and its passage through the cluster core makes it difficult to develop or retain very extended streams; instead, it possesses a system of small tidal streams much closer to the galaxy than seen in M87. The lack of tidal structures around M84 may be due to a possible recent passage through the cluster core, or M84 may simply have not experienced much recent accretion.

In contrast, both M49 and M89 display extended $(r \sim$ $50-100 \mathrm{kpc}$ ) shell systems, arguing that they have not experienced the strong tidal forces of the Virgo cluster core in the past Gyr or so. As the most luminous galaxy in the Virgo Southern Extension, it may be the dominant galaxy of group falling into the Virgo cluster for the first time, and its shell system reflects its own accretion of a smaller satellite on a radial orbit. As M49 falls into Virgo, its shell system will be disrupted and incorporated into the general ICL of the cluster. The complexity of M89's shell system argues not for an individual accretion event, but rather for multiple satellite accretions or a major merger in its past. Its system of tails, plumes, and shells will likely also be dispersed into Virgo's extended ICL as the galaxy orbits within the cluster environment.

Ultimately, however, morphology alone contains only limited information. A better understanding of these features and how they relate to the dynamical history of Virgo and its galaxies would come through studies of their kinematics and stellar populations. Kinematic information can come from identification and follow-up spectroscopy of planetary nebulae (e.g., Arnaboldi et al. 2004, Doherty et al. 2009) and globular clusters (e.g., Côté et al. 2003; Hwang et al. 2008; Lee et al. 2010) associated with the streams, while stellar populations can be studied using deep multiband surface photometry (Rudick et al. 2010) or Hubble Space Telescope imaging of resolved stars in the streams (e.g., Williams et al. 2007). Such studies would give an integrated picture of the accretion and stripping processes at work in the Virgo Cluster.

We thank Charley Knox for his tireless work on the mechanical, optical, and electronic support of the Burrell Schmidt. Over the course of this project, JCM has been supported by the NSF through grants AST9876143, ASTR-0607526, and AST-0707793, as well as by Research Corporation through a Cottrell Scholarship. CR was supported for part of this work by the Jason J. Nassau Graduate Fellowship fund.

Facility: CWRU:Schmidt

\section{REFERENCES}

Arnaboldi, M., Gerhard, O., Aguerri, J. A. L., Freeman, K. C., Napolitano, N. R., Okamura, S., \& Yasuda, N. 2004, ApJ, 614, L33

Binggeli, B. 1999, in Proc. Workshop on The Radio Galaxy Messier 87, Vol. 530, ed H.-J. Meisenheimer \& K. Röser (Berlin: Springer), 9

Binggeli, B., Tammann, G. A., \& Sandage, A. 1987, AJ, 94, 251

Bohringer, H., Briel, U. G., Schwarz, R. A., Voges, W., Hartner G., \& Trumper, J. 1994, Nature, 368, 828

Bullock, J.S., \& Johnston, K. V. 2005, ApJ, 635, 931

Canalizo, G., Bennert, N., Jungwiert, B., Stockton, A., Schweizer F., Lacy, M., \& Peng, C. 2007, ApJ, 669, 801

Caon, N., Capaccioli, M., \& D'Onofrio, M. 1993, MNRAS, 265, 1013

Caon, N., Capaccioli, M., \& Rampazzo, R. 1990, A\&AS, 86, 429

Conselice, C. J. 2006, ApJ, 638, 686

Cortese, L., Bendo, G.J., Issak, K. G., Davies, J.I., \& Kent, B.R. 2010, MNRAS, 403, L26

Côté, P., McLaughlin, D. E., Cohen, J. G., \& Blakeslee, J. P. 2003, ApJ, 591, 850

Coziol, R., \& Plauchu-Frayn, I. 2007, AJ, 133, 2630

Doherty, M., et al. 2009, A\&A, 502, 771

Dressler, A., \& Shectman, S. A. 1988, AJ, 95, 985

Elmegreen, D. M., Elmegreen, B. G., Chromey, F. R., \& Fine M. S. 2000, AJ, 120, 733

Feldmeier, J.'J., Mihos, J. C., Morrison, H. L., Harding, P., Kaib, N., \& Dubinski, J. 2004, ApJ, 609, 617

Feldmeier, J.J., Mihos, J.C., Morrison, H.L., Rodney, S.A., \& Harding, P. 2002, ApJ, 575, 779

Ferrarese, L., et al. 2000, ApJ, 529, 745
Gonzalez, A. H., Zabludoff, A. I., \& Zaritsky, D. 2005, ApJ, 618, 195

González-García, A. C., \& Balcells, M. 2005, MNRAS, 357, 753

Harris, W. E., Durrell, P. R., Pierce, M. J., \& Secker, J. 1998,

Nature, 395,45

Hernquist, L., \& Quinn, P. J. 1988, ApJ, 331, 682

Hernquist, L., \& Quinn, P. J. 1989, ApJ, 342, 1

Hernquist, L., \& Spergel, D. N. 1992, ApJ, 399, L117

Hibbard, J. E., \& Mihos, J. C. 1995, AJ, 110, 140

Hwang, H. S., et al. 2008, ApJ, 674, 869

Irwin, J. A., \& Sarazin, C. L. 1996, ApJ, 471, 683

Jacoby, G. H., Ciardullo, R., \& Ford, H. C. 1990, ApJ, 356, 332

Jedrzejewski, R. I. 1987, MNRAS, 226, 747

Kenney, J.D.P., Tal, T., Crowl, H.H., Feldmeier, J., \& Jacoby, G.H. 2008, ApJ, 687, L69

Kim, E., Lee, M. G., \& Geisler, D. 2000, MNRAS, 314, 307

Knebe, A., Müller, V. 2000, A\&A, 354, 761

Kormendy, J., Fisher, D. B., Cornell, M. E., \& Bender, R. 2009 ApJS, 182, 216 (K09)

Lee, M. G., Park, H. S., \& Hwang, H. S. 2010, arXiv:1003.2499

Malin, D. 1994, in IAU Symp. 161, Astronomy trom Wide-Field

Imaging, ed. H. T. MacGillivray et al. (Dordrecht: Kluwer), 567

Malin, D.F. 1979 Nature, 285, 643

Malin, D.F., \& Carter, D. 1980, Nature, 285, 643

Mathiesen, B., Evrard, A. E., \& Mohr, J. J. 1999, ApJ, 520, L21

McLaughlin, D. E. 1999, ApJ, 512, L9

Mei, S., et al. 2007, ApJ, 655, 144 
Mihos, J. C. 2004, in Carnegie Observatories Centennial Symp., Clusters of Galaxies: Probes of Cosmological Structure and Galaxy Evolution, ed. J.S. Mulchaey, A. Dressler, \& A. Oemler (Cambridge: Cambridge Univ. Press), 277

Mihos, J. C., Harding, P., Feldmeier, J., \& Morrison, H. 2005, ApJ, 631, L41

Mihos, J.C., Janowiecki, S., Feldmeier, J.J., Harding, P., \& Morrison, H. 2009, ApJ, 698, 1879

Miville-Deschênes, M.A., \& Lagache, G. 2005, ApJS, 157, 302

Morrison, H.L., Miller, E.D., Harding, P., Stinebring, D.R., \& Boroson, T.A. 1997, AJ, 113, 2061

Oegerle, W. R., \& Hill, J. M. 2001, AJ, 122, 2858

Patterson, R. J., \& Thuan, T. X. 1992, ApJ, 400, L55

Peletier, R. F., Davies, R. L., Illingworth, G. D., Davis, L. E., \& Cawson, M. 1990, AJ, 100, 1091

Quinn, P. J. 1984, ApJ, 279, 596

Rudick, C. S., Mihos, J. C., Frey, L. H., \& McBride, C. K. 2009, ApJ, 699, 1518

Rudick, C. S., Mihos, J. C., Harding, P., Feldmeier, J.J., Janowiecki, S., \& Morrison, H.L. 2010, in preparation
Rudick, C. S., Mihos, J. C., \& McBride, C. 2006, ApJ, 648, 936 Sandage, A. 1976, AJ, 81, 954

Schindler, S., Binggeli, B., Böhringer, H. 1999, A\&A, 343, 420

Schlegel, D. J., Finkbeiner, D. P., \& Davis, M. 1998, ApJ, 500, 525

Schweizer, F. 1982, ApJ, 252, 455

Seigar, M. S., Graham, A. W., \& Jerjen, H. 2007, MNRAS, 378, 1575

Stickel, M., Bregman, J. N., Fabian, A. C., White, D. A., \& Elmegreen, D. M. 2003, A\&A, 397, 503

Tal, T., van Dokkum, P. G., Nelan, J., \& Bezanson, R. 2009, AJ, 138,1417

Toomre, A., \& Toomre, J. 1972, ApJ, 178, 623

Weil, M. L., Bland-Hawthorn, J., \& Malin, D. F. 1997, ApJ, 490, 664

West, M. J., Jones, C., \& Forman, W. 1995, ApJ, 451, L5

Williams, B. F., et al. 2007, ApJ, 656, 756

Witt, A. N., Mandel, S., Sell, P. H., Dixon, T., \& Vijh, U. P. 2008, ApJ, 679, 497

Zaritsky, D., \& Rix, H.-W. 1997, ApJ, 477, 118 